\title{
Best Practices for Working with Non-Technical Project Sponsors
}

\section{Dr. John Paul Farris, Grand Valley State University}

John Farris joined the faculty at Grand Valley State University after a successful tenure as the chief product designer for a medical device manufacturer. His other significant industrial experience includes designing engine components for Caterpillar Inc. and consulting on the design of stationary fuel cell power generation units. His current research interests are design methods and medical technology. He received his Ph.D. from the University of Rhode Island and his Bachelors and masters degrees from Lehigh University.

\section{Dr. Wendy S. Reffeor, Grand Valley State University}

Wendy Reffeor is an Associate Professor of Mechanical Engineering in the Padnos College of Engineering and Computing at Grand Valley State University. She earned her Bachelors from GMI Engineering \& Management Institute, Masters from Purdue University and Doctorate from Michigan State University. Her industrial experience includes designing quality systems for Allison Engine Company in Indianapolis. Since joining GVSU, she has focused on introducing design and build projects in traditionally analytical courses in the Engineering Mechanics sequence.

\section{Dr. Lisa Karen Kenyon, Grand Valley State University}

Dr. Kenyon is a pediatric physical therapist and Associate Professor in the Department of Physical Therapy at Grand Valley State University in Grand Rapids, Michigan. 


\section{Best Practices for Working with Non-Technical Project Sponsors}

\section{Abstract}

Spurred on by the growth of project-based learning, experiential learning and service learning, many engineering programs now have engineering students working on projects sponsored by non-technical sponsors. In addition many teams include students from disciplines other than engineering. Mentoring a team of students working with non-technical project sponsors and team mates is much different than mentoring a team of engineering students working on a project with a practicing engineer serving as a sponsor. Non-technical students and sponsors do not understand the engineering design process, engineering analysis and the culture in engineering. Engineering students and faculty may know about the technology available but they may have little understanding of a problem involving other disciplines. In addition these problems may be complex in nature and involve stake holders with conflicting priorities. Finally, the project sponsors and experts with domain specific knowledge may not have the time, ability or motivation to teach engineering students and faculty the basic concepts about their discipline or problem. Communication between project stakeholders is furthered hampered by the different frames of reference, experiences, lack of common disciplinary knowledge and the tacit or unspoken knowledge of all stake holders.

Similarly, engineering students and faculty struggle to understand the cultural norms of the sponsor's profession and the priorities of the stakeholders. Some sponsors bring potential solutions to the table and can be disappointed when the design team recommends implementing other technologies. These challenges confront faculty sponsors as they try to mentor teams and satisfy all stakeholders. Ideally, the students should have a positive learning experience, the sponsor should value the final output of the project and faculty should not have to spend too much time supervising the project. Effective communication between the stakeholders from different disciplines combined with managing expectations is the best way to improve the chances of success. This paper will step through the design process and describe the best practices for facilitating communication between the sponsor, engineering students and faculty related to each step of the design process. Completed Projects are used as examples to illustrate the difficulties.

Introduction

Over the past 20 years, there has been a steady increase in the interest in project based and service learning as can be seen in Figure 1 (ASEE Conference Proceedings Search, 2017) below. Over those twenty years, the number of papers presented on service learning has increased by 8.4 times and those on project based learning by 6.7 times. 


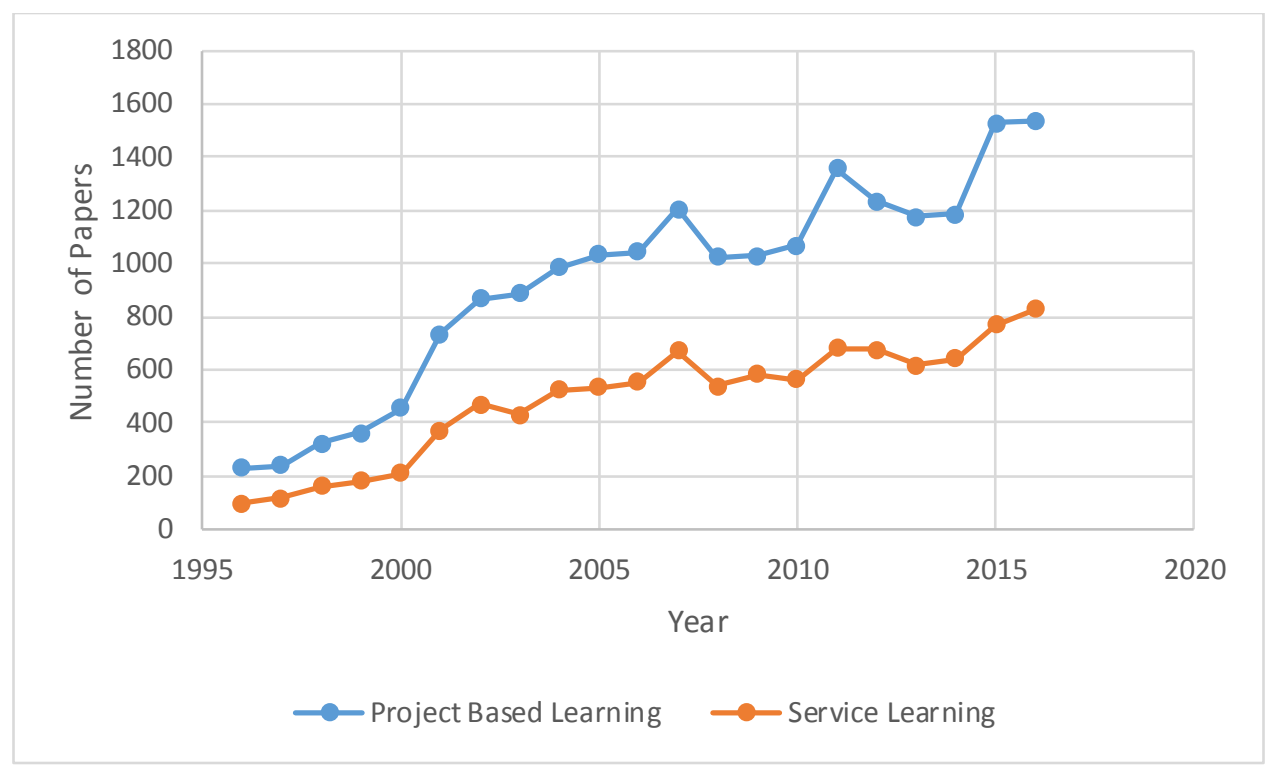

Figure 1. Number of Papers at ASEE Annual Conference and Exposition by Year

Additionally, in 2006 the International Journal for Service Learning in Engineering, was founded. This journal is exclusively devoted to publishing works on the impact of service learning in engineering education. One issue in particular, Special Issue: University Engineering Programs That Impact Communities: Critical Analyses and Reflection, focused on community impact. Schools such as

This increase in project based and service learning has led to a need for students engaged in these projects to understand and address stakeholders who do not have a technical background.

Zoltowski and Oakes (Carla B. Zoltowski, 2014) discuss this need as well as the need to develop and maintain relationships with community partners. Additionally, the difficulty of understanding conflicting priorities of project sponsors is addressed by Malkin and Calman (Robert Malkin, 2014). Finally, a method to assess needs of individuals in developing countries and persons with disabilities, "Contextual Needs Assessment" was developed (Norton, 2008).

In this paper, a methodologies for addressing the multiple concerns of working with nontechnical project sponsors is addressed. The papers organized around a generic model for the engineering design process shown in Figure 2.

\section{Gathering information phase}

The better students understand the problem the more likely their solution will solve the problem. Unfortunately, asking students to go out and gain an understanding of the problem without guidance is not terribly effective. In 2005 Matthew Green (Mathew Green, 2005) developed a method to address this problem as part of his $\mathrm{PhD}$ dissertation at the University of Texas, Austin. He followed up his dissertation by presenting a paper that assessed his proposed methodology at 
the national conference of the American Society for Engineering Educators (Green, 2006). Green broke down the context of a product into four distinct categories:

1. How the product will be used?

2. Where the product will be used?

3. Who will use the product?

4. What competitive products are already on the market?

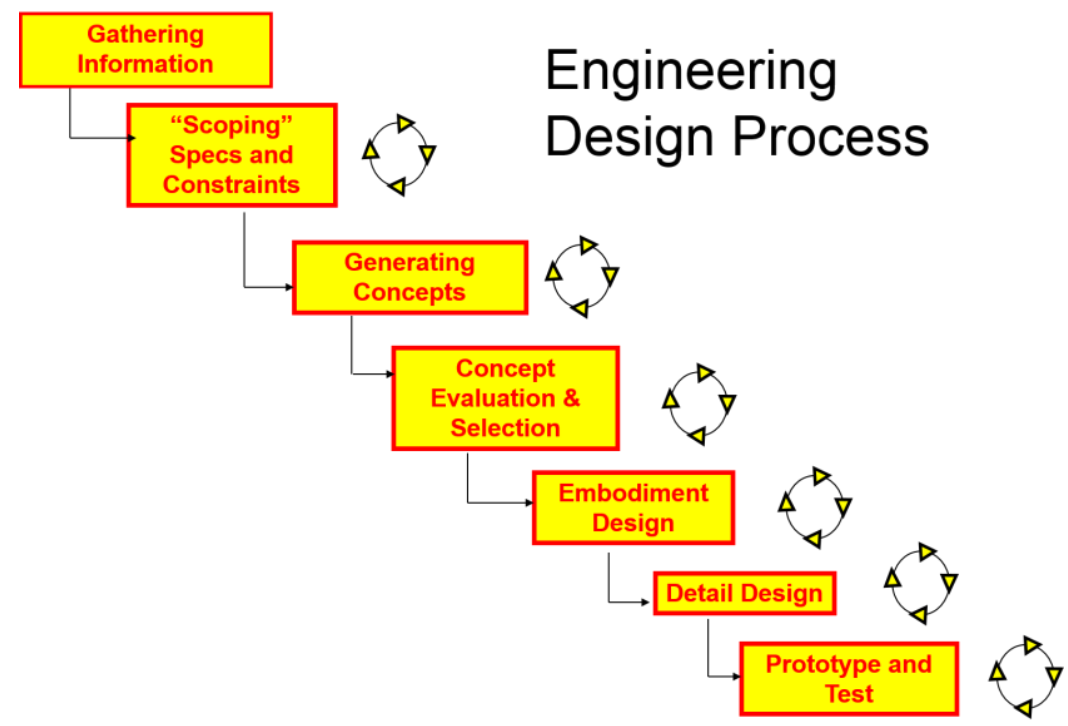

Figure 2. A generic model for the engineering design process.

To follow Green's methodology students first brainstorm questions for potential users and other stakeholders and then they compare their answers to a template provided by Green to determine if the team has overlooked any important questions.

In addition Green and his colleagues have developed a list of questions that a design team developing a new pencil sharpener might ask potential customers and other stakeholders. By studying the example and following the method students are much more likely to discover allimportant aspects of the products context that will impact the design. In 2016 a team of junior engineering students at a Midwestern university took on the challenge of designing a better way to move patients from a bed to a stretcher and back again. The current method that dates back to World War I requires at least 3 caregivers. The current method can and does cause serious and expensive musculoskeletal injuries to nursing staff and lost productivity. The average age of a hospital nurse is 47 years. Statistics from the National Institute of Occupational Safety and Health and the Centre for Disease Control show that these workers have musculoskeletal injury rates greater than construction and manufacturing industries. Sprains and strains account for more than half of all hospital work-related injuries and the cost to the US health care industry is estimated to be in excess of 20 billion dollars annually. In response to this problem, hospitals 
have purchased and installed a variety of devices ranging from expensive overhead lifts to slippery sheets of material intended to reduce the force of friction. However, user acceptance of these devices remains very low. One nurse answered a student's question about competitive devices by saying "We never use the overhead lifts because they take too much time to use." The lack of acceptance of the new devices may be attributed to the designers' lack of understanding of the context in which their products operate. Nurses are pressed for time and will put themselves at risk to save time. The students understood that their design must be quick to set up and use. In addition they could estimate how quick by understanding the time required to set up and use competitive devices. This insight was formalized in the specifications for the device.

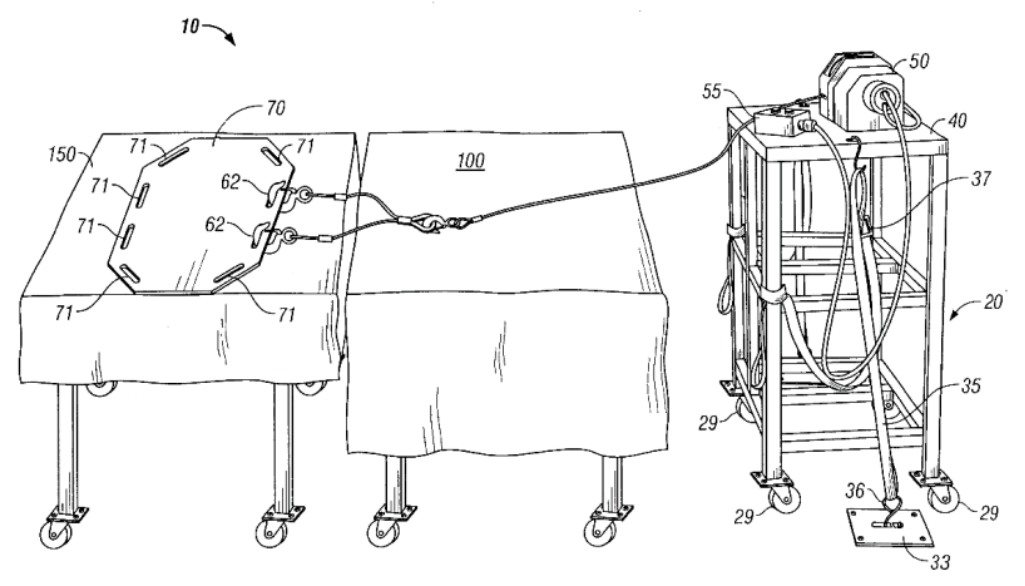

Figure 3. Drawing from US patent number US6728979 B1

Similarly, the device shown in figure three has one flaw that may have prevented the device from being commercialized. The inventor did not understand the "where" context of the problem. Specifically he or she did not understand that the device would be used in a hospital room crowded with medical equipment and short on storage space. The size of the device may be why the owner of the patent decided not to pay the patent fees. Therefore a team of students using Green's methodology required their design to be small enough to be stored under the existing stretcher. Again, a specification was created to formalize this insight.

Scoping, specifications, and constraints phase

The author's teach the use of the House of Quality methodology (Hauser, R, 1988) to convert customer quotes into specifications for a product. Before beginning design activities it is common to have the sponsor approve the specifications that the design team has formulated. If the sponsor is familiar with the design process and has a technical background this is not a challenge. However, for non-technical sponsors a simple list of specifications may not communicate the intent of the design team well enough to allow the sponsor to give meaningful feedback and, hopefully, head off any problems early in the design process. For better communication, two methods are recommended. First, the design team should communicate how the specifications will be used in the design process. It is important for sponsores to realize that 
the specifications are used to guide the design. They will be used to reject concepts that cannot meet the specifications, and for the selection of appropriate off-the-shelf components for incorporation into the design. Specifications also have a direct impact on the cost of the design. Therefore it is important for the sponsor to fully understand each specification and to ensure that all functionality of the proposed product is included in the specifications. If it is not in the specifications product will not do it.

Next, the sponsors need to be able to relate to the quantities that are used in the specifications. For instance, a student design team working on a minimally invasive surgical tool was trying to agree on the limits of the radius of curvature for the device. The surgeon was having a hard time determining the minimum and maximum acceptable radius for the device. To resolve the situation, the students printed out a series of increasing radii for the surgeon to examine. By referring to the $\mathrm{CAD}$ printouts, the surgeon was then able to confidently quantify acceptable limits for the device.

Similarly, when a team of students were designing an adjustable head array to allow children with special needs to practice using power mobility, the question of how large a force should be required to activate the switch. A head array consists of three switches arranged on each side and in back of the child's head. Children who have best control of their head movements use these devices to control power wheelchairs and other devices. A picture of the finished device can be seen in figures four and five. The physical therapists sponsoring the project desired a head array that could be adjustable in two ways. They requested the ability to adjust the position of each switch, and the force required to activate each switch. The desired distance of adjustability for the position of each switch was relatively easy to quantify with a tape measure. However, the adjustability of the force required and the smallest increment of that adjustability was difficult for the physical therapists to specify. After much discussion and more than one meeting, the students presented the physical therapists with a collection of weights that they used to judge the appropriate values for the specifications dealing with the force required to activate the switch. Later in the design process students refined the limits in the specifications by creating a focus prototype of the buttons and measuring force applied by the physical therapists when they use the button. The final specification stated that the buttons have an activation force varying between 2.5 ounces and 6 ounces with a minimum increment of half an ounce. 


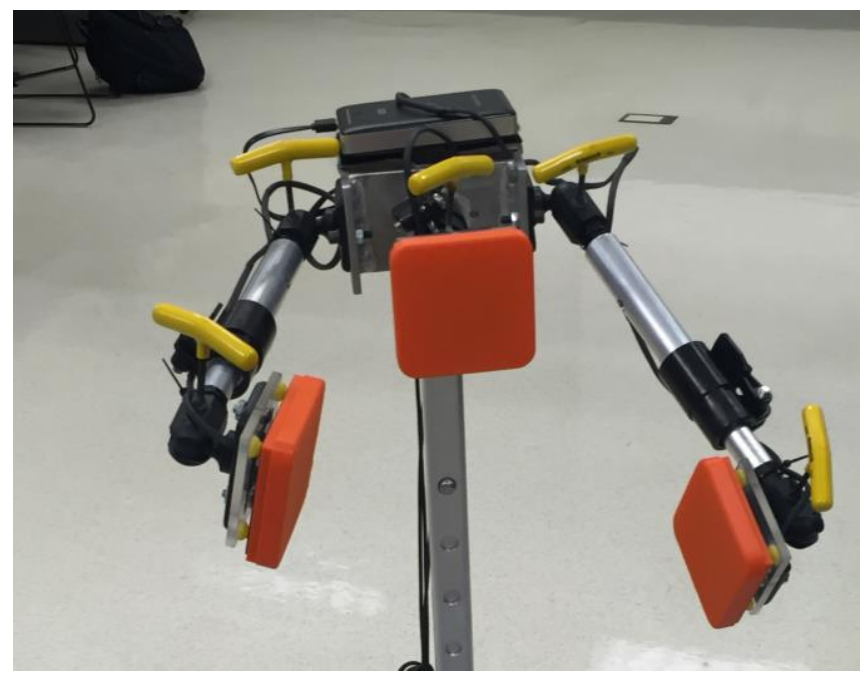

Figure 4. The adjustable head array with 3 buttons corresponding to moving a power wheelchair left, right and forward.

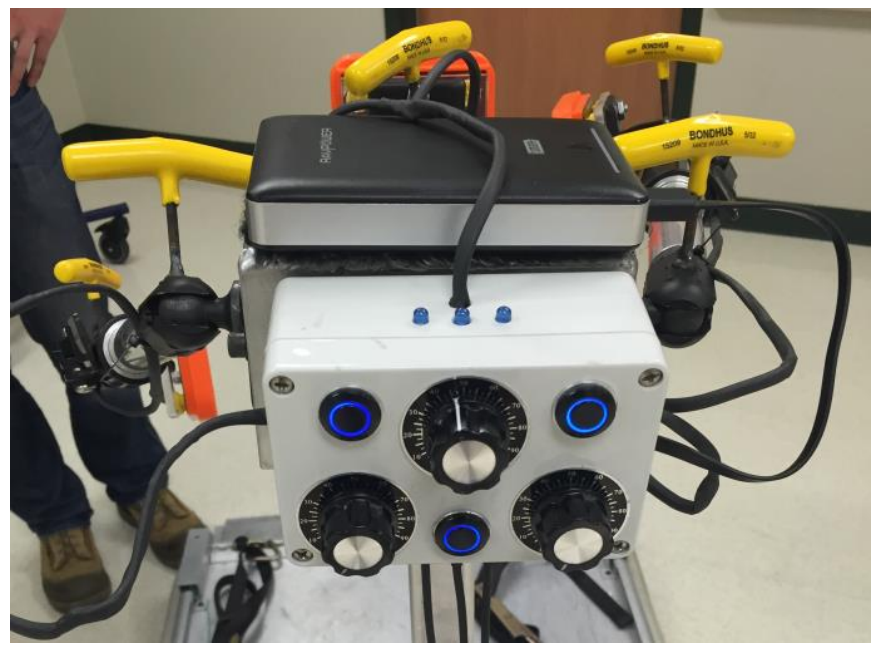

Figure 5. The controls for the adjustable head array. The dials adjust the force required to activate the switch and the lighted buttons indicate if the switch is active.

Another method to help non-technical sponsors understand specifications so that they can critique the specifications is the use of visual specifications. Visual specifications use a mockup of the proposed product shown in products intended use environment to justify the target values of specifications and show how the specification relates to the use of the product. 


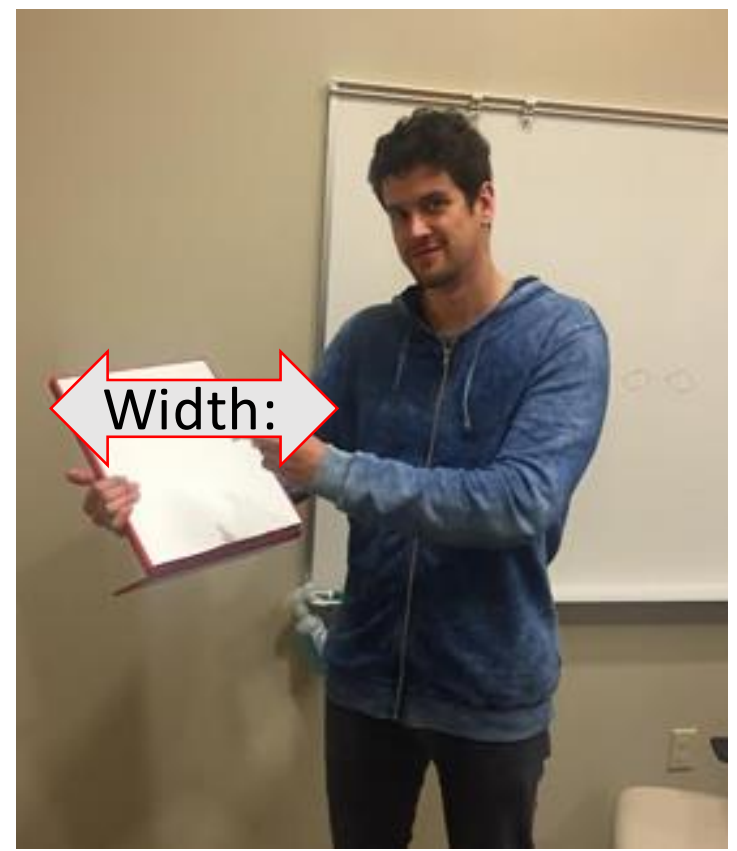

Figure 6 A design team member demonstrating will the work sharing use case and the impact of the width specification.

Examples of visual specifications are shown in Figures 6 and 7. The product in the figures is a personal white board designed for classroom use to facilitate individual and collaborative student work. In this example a team composed of business students, engineering students and industrial design students was working to lower the cost of the product, refine the use cases for the product and develop the target market for the product.

Figures 6 shows a student demonstrating how a user might use the product to share his or her work with a small group of colleagues. By labeling width of the board in the picture, the picture shows how the width specification impacts the use scenario.

Similarly Figure 7 shows the impact of the products height specification on the individual work use case. 


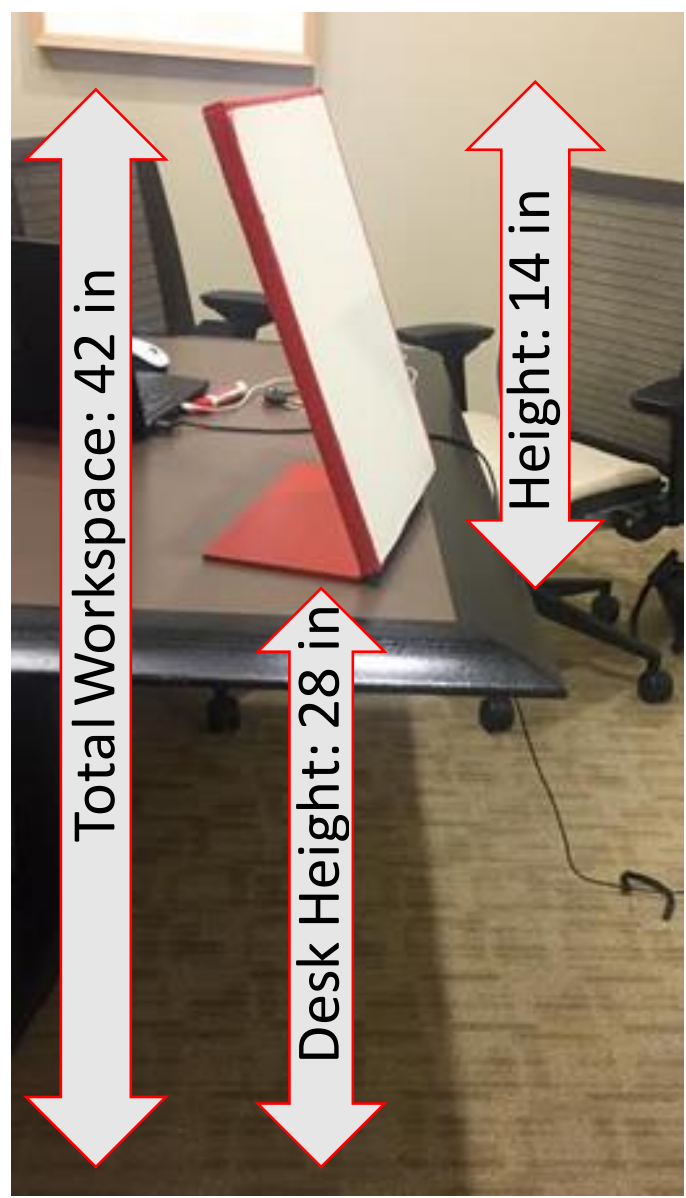

Figure 7. The impact of the height specification on the individual work is case.

Concept generation phase

The intent of the concept generation effort is to develop a diverse set of concepts that can be compared, combined and refined into the best concept to solve the particular problem. The phase starts with a completed Function Structure Diagram (FSD). A FSD encodes the students understanding of the problem as well as assumptions that have been made about the solution. The authors employ many different, but conventional, methods to spur of the students to generate ideas. Most methods yield ideas for solving part of the problem. Morphological matrices that contain the critical sub functions from the FSD will are an efficient way record the results of the students' ideation efforts. In addition, all stakeholders should be interviewed to elicit ideas they have for solving the problem. However, students often want to develop ideas suggested by stakeholders for two reasons. First, stakeholders are often viewed as experts in the field and students are often hesitant to challenge their ideas. Also, students often believe that their grade is tied to satisfying the stake holders. This tendency can be overcome by decomposing stakeholders' ideas into solutions for critical sub functions and entering the solutions into the morphological matrix. Then when students go to create concepts by selecting solutions for each critical sub functions from the morphological matrix, stakeholder solutions receive the same 
amount of attention as ideas developed by other methods. In addition the authors strongly encourage design teams to develop many different conceptual designs to be processed using the Pugh selection method (Pugh 1990).

Concept evaluation and selection phase

The selection of the most appropriate concept to pursue is an important decision that is critical to the success of a project. A poor choice leads to wasted time and effort and risks reducing the quality of the final prototype. In addition, input from the sponsor and other stakeholders can have the greatest impact on the success of the project during this phase. However, successful strategies for eliciting and incorporating sponsor feedback must take into account that most sponsors and stakeholders have limited time to devote to the project. In addition, student teams also must move quickly and efficiently if they are to complete projects on time. Therefore, any system should minimize students' time and effort spent developing concepts that will ultimately be discarded. The authors have developed and deployed a three phase approach to gather the most important feedback as early as possible with as minimum of time required from the stakeholders.

In the first phase of review the students use hand sketches and voice over PowerPoint videos to solicit feedback from stakeholders. Students to use the Pugh concept selection process to select and refine the three concepts with different operating principles. Next, the students create a series of sketches that describe how each concept operates. The sketches are used to create a short voice over PowerPoint presentation that is posted on YouTube, with the appropriate privacy settings, for the stakeholders to view. Videos must be less than 2 minutes long. After viewing each video, stakeholder is asked the following questions:

1. What do you like about this concept?

2. What do you not like about this concept?

3. What has not considered in this concept?

Posting the videos on YouTube allows stakeholders to easily view the videos at their own convenience. Students can quickly create these presentations with their phone or by using presentation software like PowerPoint. Sketches with a verbal description have proven to be a much better way to communicate the design when compared to sketches alone. Figures 8 and 9 show examples of sketches students have used to communicate concepts to stakeholders. As you can see, the sketches need to be clear but do not have to be beautiful in order to communicate effectively. The quantity and quality of sponsor feedback has improved dramatically since the sketches and voice over PowerPoint approach has been implemented. 


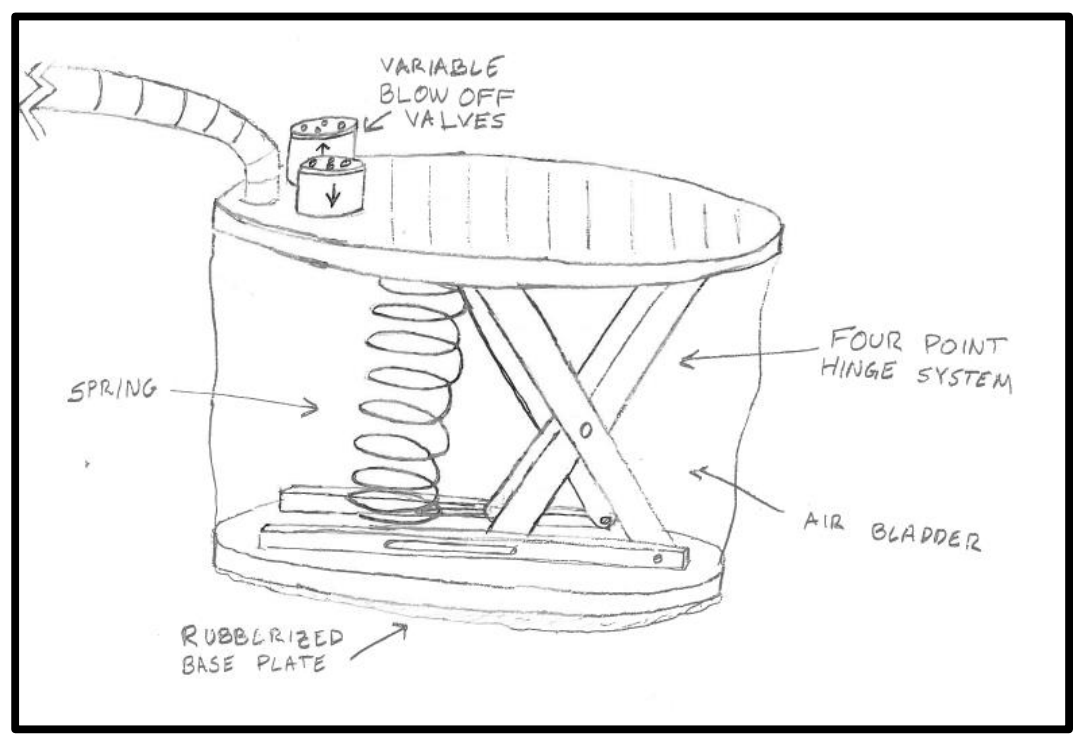

Figure 8. An example of a sketch used to describe a concept for a medical device.

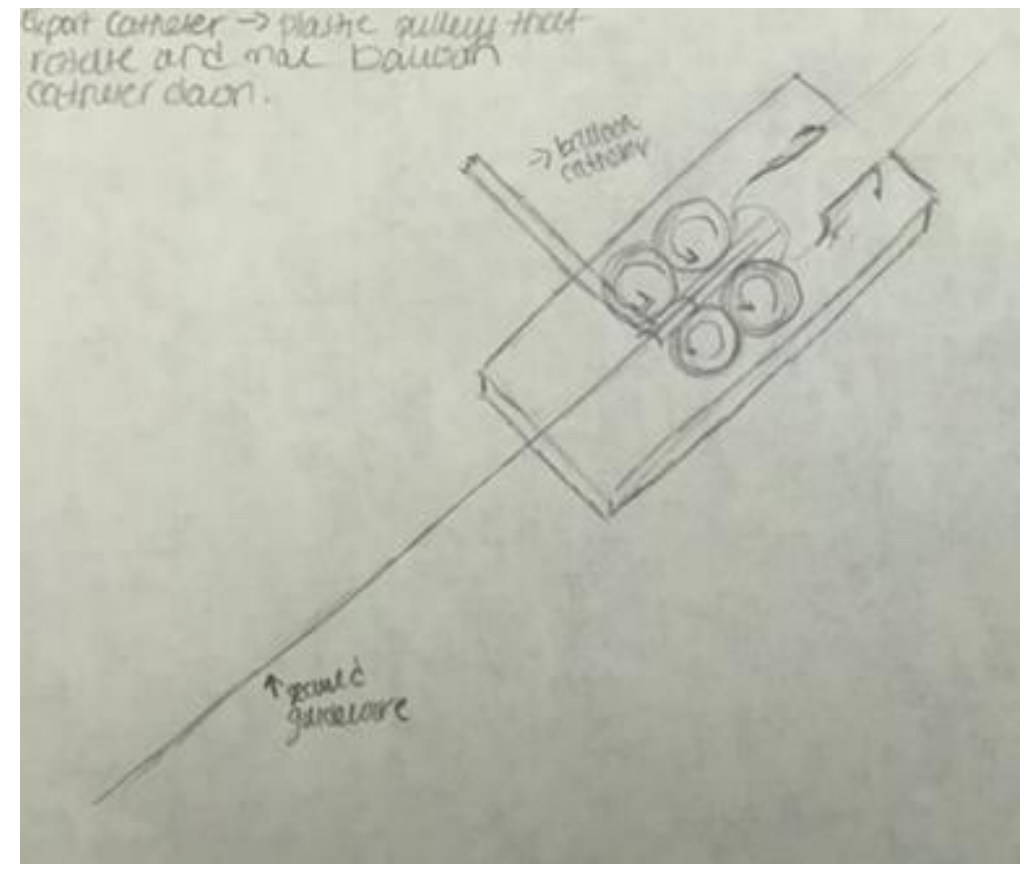

Figure 9. An example of a sketch used to describe a concept.

In the second phase of the approach, students analyze the answers to the questions provided by the stakeholders, eliminate clearly inferior concepts, and refine concepts still under consideration. Mockups of viable concepts are created using materials that are readily available, inexpensive and easy to work with such as cardboard, foam board and clay. The mockups are used to create videos that illustrate the use case for the product. Again these short videos are used to elicit feedback from stakeholders and stakeholders are asked the same questions:

1. What do you like about this prototype? 
2. What do you not like about this prototype?

3. What has not considered in this prototype?

Figure 10 shows a mockup of a cough assist product used in a video to elicit stakeholder

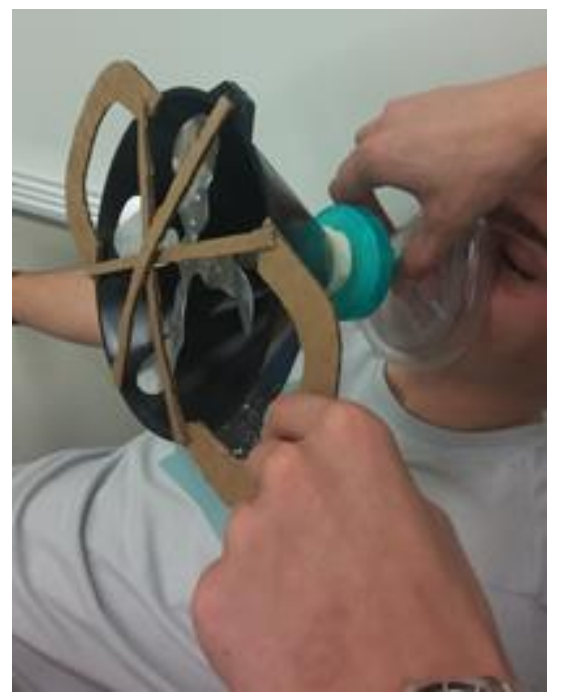

Figure 10. A mock up used in a video to illustrate the use case for a cough assist product.

In the third phase students analyze feedback from stakeholders and eliminate concepts from consideration. Generally, student teams settle on one concept to move forward. Then a "works like" prototype is built for the concept selected. "Works like" prototypes are rough, unrefined, ugly prototypes that are used to address the largest risk associated with the proposed concept. The issue should be the most risky technology employed or the most severe usability concern raised by the stakeholders. "Works like" prototypes must be refined enough to address most urgent concerns of the design. All other aspects of the prototype should be designed so that the team can get the prototype in front of the stakeholders as soon as possible. Strategies for building "works like" quickly include using frame systems like 80/20, cannibalizing existing products for parts and assemblies, and using a computer-controlled manufacturing process like rapid prototyping, laser cutting and CNC machining. "Works like" prototypes do not meet all specifications. These prototypes are louder, heavier and harder to use than the intended product. For example, a "works like" prototype for a patient moving system consisted of a frame made from $80 / 20$, a winch used to move cars, locking pliers commonly used in welding operations, and a few laser cut parts. This "works like" prototype was fabricated in one week.

Stakeholder reviews of "works like" prototypes is best done in face-to-face. Ideally, stakeholders are able to use the "works like" prototype in conditions as close to possible as the conditions of product will be used in. However, students should be warned that stakeholders have an uncanny ability to break prototypes. Therefore, video should be taken before stakeholders use any prototype. A physical prototype elicits valuable feedback from stakeholders and, often, keeps them excited and engaged in the process.

Embodiment design phase 
After the concept to pursue have been identified, it is very important to manage the sponsor's expectations in regards to cost and functionality. Before beginning the construction of the final prototype a bill of materials is created in order to estimate the cost of the project. The scope of the final prototype is also defined and presented to the sponsor for their approval. In this context the scope includes the planned functionality of the prototype as well as the intended use of the prototype. For instance a prototype may be built to answer a technical feasibility question. Another reason to build a prototype might be to gather more user feedback. Finally, some prototypes will be put to use by the sponsor. Many assistive technology prototypes will immediately be put to use. The purpose of the prototype impacts the cost and time required to create the prototype.

During the design refinement phase, students predict the performance of the prototype using analytical models and conduct experiments to prove the feasibility and performance of the prototype. When analytical or experimental results are obtained that force changes in the functionality of the design or the cost of the project, sponsors should be informed as soon as possible. Ideally students will formulate alternative paths to compensate for the unexpected results and present the alternative to the sponsor. As usual, students benefit from creating arguments to recommend one alternative in a set of feasible alternatives. For example, one team had pursued a concept involving nitinol wire. Nitinol wire was chosen for its shape changing abilities. Unfortunately, the surgeon sponsoring the project insisted that the students change the design to use a larger diameter of wire. This change resulted in the recovery strain limit of the Nitinol wire to be exceeded. The student team was forced to resurrect other concepts for consideration and the delivery date for the prototype was delayed.

The role of iteration

The role of iteration in the improvement and refinement of products must be clearly communicated to sponsors in order to manage their expectations. Often, the use of the first prototype built will elicit additional design issues that must be addressed. If the new issues are extensive enough they may serve as the basis for another student project. Non-technical sponsors who are not familiar with the necessity for iteration need to be made aware of possibility that another iteration will be required to develop prototype that fully meets their needs. When the iteration is performed there is also the opportunity to include new functionality into the prototype.

The evolution of design of a hugging chair intended to be used to calm children with special needs illustrates the benefits of iterating. Hugs are used to calm children with special needs. A physical therapist working with this population desired a chair that would apply gentle pressure to the upper arms of student while the student engaged in academic activities in the classroom. Figure 11 shows the original design of the hugging chair. The original design employed a rope and pulley system to allow the student to apply the desired amount of pressure. However, the design was bulky, complicated, and unreliable. When the chair was deployed classroom it was discovered that many children did not have the strength or coordination to generate the desired pressure. In addition, sharp corners in the design were a danger to children. 


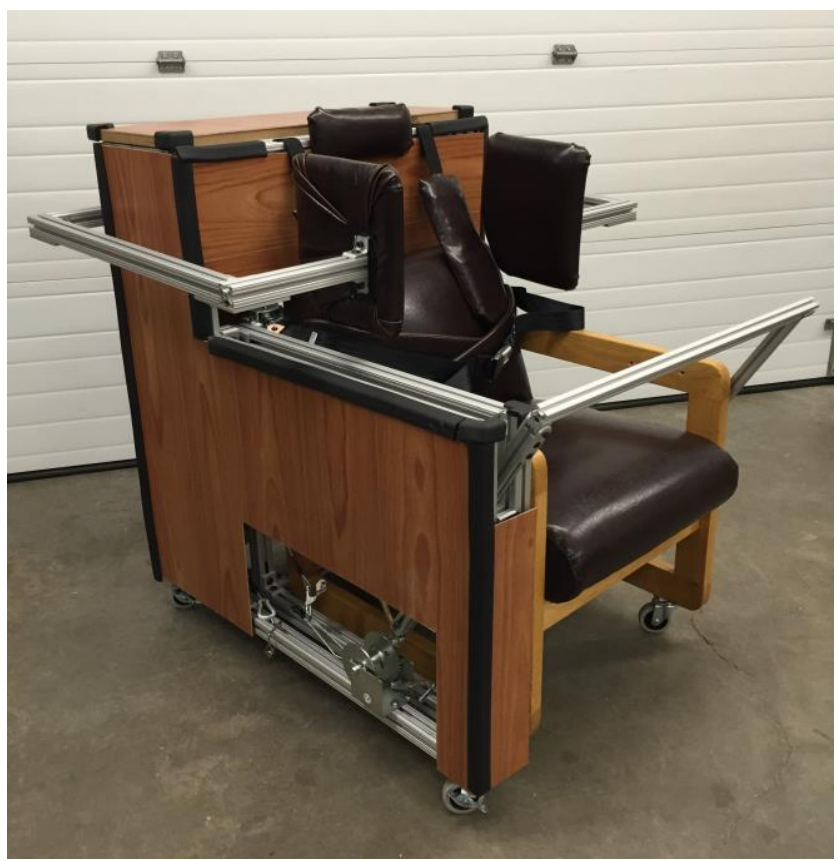

Figure 11. The original designs of the hugging chair.

A second design team was assigned to address these concerns. The first iteration of the chair is shown in Figure 12. The new design uses an electrically powered linear actuator to allow

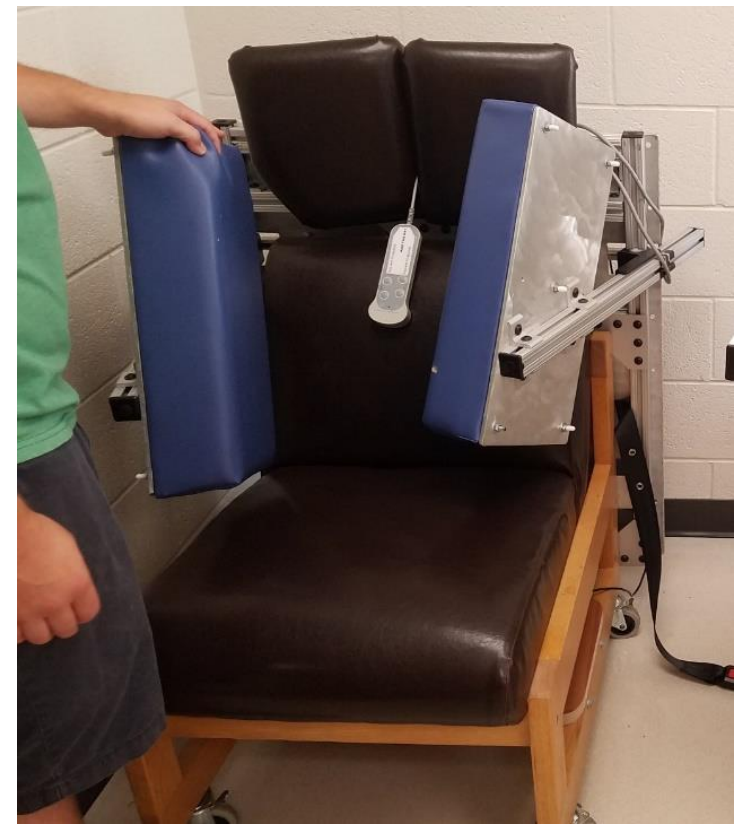

Figure 12. The first iteration of the hugging chair.

the user to control the magnitude of force applied. In addition, the elimination of the crossbar in front of the chair, made the design much easier for children to use. The size of the chair was reduced making it easier to transport and the new design looked more like a regular chair. 
Unfortunately users complained that the mechanical system did not deliver a realistic feeling hug.

Compressed air was used in the second iteration of the hugging chair, shown in figure 13 , to provide a more realistic feeling hug. A small compressor is located underneath the seat and a pressure regulator allows the user set the desired pressure. The new design is small, easily portable and perceived as desirable by all students in the class.

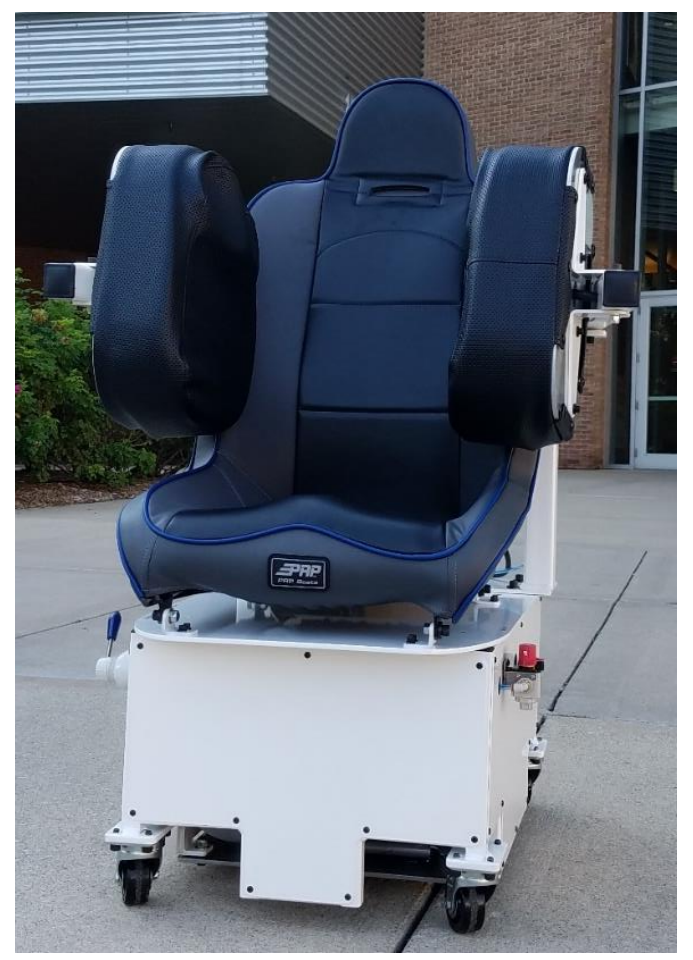

Figure 13. The second iteration of the hugging chair.

Discussion

The root cause of many of the challenge discussed in this paper can be traced back to poor communication and a failure to manage expectations. The differences between engineering and other professions in educational background, experience and workplace norms makes communication more difficult. In addition, the literature shows that engineering students' communication skills do not meet the expectations of employers. (N.Spinks, 2008) (Ronald Meier, 2000) The authors experience and the literature suggest that a targeted interventions focused on preparing the students to communicate with people with non-technical backgrounds may improve the students' performance on the design teams and help them succeed in industry. Ideally this intervention would build on the students' previous training in technical communication and be designed to fit into a full class syllabus.

Managing the sponsors' expectations is primarily the responsibility of the faculty. Along with frequent communication from the faculty keeping the sponsor informed about the progress of the team and sponsor action required, professors with much experience with guiding student design projects recommend that all sponsors read and sign an agreement that states the expectations for 
the project. These expectations include information about any financial obligations, meetings sponsors must attend, the total time commitment required, and expected outcomes of the project.

\section{Conclusion}

The advantages of working with non-technical sponsors are significant and include interesting, challenging projects for students, involvement with local industry and community, and the opportunity for students to improve their communication skills by explaining technical concepts to non-engineers. However, care must be taken to ensure a satisfactory outcome for students and all stakeholders. This paper has suggested several techniques that can be used to make these types of projects more successful.

\section{References}

ASEE Conference Proceedings Search. (2017, February 1). Retrieved from https://peer.asee.org/: https://peer.asee.org/

Carla B. Zoltowski, W. C. (2014). Learning by Doing: Reflections of the EPICS Program. International Journal for Service Learning in Engineering, 1-32.

Green, M. G., 2005, "Enabling Design in Frontier Contexts: A Contextual Needs Assessment Method with Humanitarian Applications," PhD Dissertation, Mechanical Engineering, University of Texas, Austin.

Green, M.G., Linsey, J., Seepersad, C., Schmidt, K., and Wood, K., 2006, "Design for Frontier Contexts: Classroom Assessment of a New Design Methodology with Humanitarian Applications", American Society of Engineering Educators Conference.

Meier, R. L., Williams, M. R., \& Humphreys, M. A. (2000). Refocusing our efforts: Assessing non-technical competency gaps. Journal of Engineering Education, 89(3), 377.

Pugh, S., 1990, Total Design: Integrated Methods for Successful Product Engineering, Pearson Education Limited, Essex, England.

Hauser, R., Clausing, D., (1988). The House of Quality, Harvard Business Review, 63-73.

Robert Malkin, L. J. (2014). Service-Learning in Biomedical Engineering: Engineering World Health (EWH). International Journal for Service Learning in Engineering, 39-47.

Spinks, N., Silburn, N. L.J. and Birchall, D. W. 2008. Making it all work: the engineering graduate of the future: a UK perspective. European Journal of Engineering Education, 32(3): $325-335$. 